\title{
Single Carrier Modulation With Nonlinear Frequency Domain Equalization: An Idea Whose Time Has Come - Again
}

\author{
In high-speed single-carrier digital communication systems, \\ processing blocks of signals using Fast Fourier Transforms is an efficient way to \\ equalize (compensate) for interference between transmitted symbols.
}

By Nevio Benvenuto, Senior Member IEeE, Rui Dinis, Member IEeE, David Falconer, Life Fellow IEEE, and Stefano Tomasin, Member IEEE

\begin{abstract}
In recent years single carrier modulation (SCM) has again become an interesting and complementary alternative to multicarrier modulations such as orthogonal frequency division multiplexing (OFDM). This has been largely due to the use of nonlinear equalizer structures implemented in part in the frequency domain by means of fast Fourier transforms, bringing the complexity close to that of OFDM. Here a nonlinear equalizer is formed with a linear filter to remove part of intersymbol interference, followed by a canceler of remaining interference by using previous detected data. Moreover, the capacity of SCM is similar to that of OFDM in highly dispersive channels only if a nonlinear equalizer is adopted at the receiver. Indeed, the study of efficient nonlinear frequency domain equalization techniques has further pushed the adoption of SCM in various standards. This tutorial paper aims at providing an overview of nonlinear equalization methods as a key ingredient in receivers of SCM for wideband transmission. We review both hybrid (with filters implemented both in time and frequency domain) and all-frequency-domain iterative struc-
\end{abstract}

Manuscript received March 25, 2008; revised January 29, 2009. Current version published December 23, 2009.

N. Benvenuto and $\mathbf{S}$. Tomasin are with the Department of Information Engineering, University of Padova, Italy (e-mail: nb@dei.unipd.it; tomasin@dei.unipd.it).

R. Dinis is with IT (Instituto das Telecomunicações) and FCT-UNL

(Faculdade de Ciências e Técnologia da Universidade Nova de Lisboa), Lisbon, Portugal (e-mail: rdinis@ist.utl.pt).

D. Falconer is with the Department of Systems and Computer Engineering, Carleton University, Ottawa, Canada (e-mail: ddf@sce.carleton.ca).

Digital Object Identifier: 10.1109/JPROC.2009.2031562 tures. Application of nonlinear frequency domain equalizers to a multiple input multiple output scenario is also investigated, with a comparison of two architectures for interference reduction. We also present methods for channel estimation and alternatives for pilot insertion. The impact on SCM transmission of impairments such as phase noise, frequency offset and saturation due to high power amplifiers is also assessed. The comparison among the considered frequency domain equalization techniques is based both on complexity and performance, in terms of bit error rate or throughput.

KEYWORDS | Decision-feedback equalizers; digital modulation; discrete Fourier transforms; multiple antennas

\section{INTRODUCTION}

Equalization - the compensation of the linear distortion caused by channel frequency selectivity - is an essential component of digital communications systems whose data symbol rate is higher than the coherence bandwidth of typically encountered channels. Intersymbol interference that afflicts serial data transmission has traditionally been mitigated by equalization implemented in the time domain with linear filtering, usually with a transversal structure, hence the designation linear equalizer [1]. Due to the tradeoff between equalization of the channel impulse response to remove intersymbol interference (both precursors and postcursors) and noise enhancement at the decision point, a linear equalizer yields less than ideal performance in terms of bit error rate, especially in 
dispersive channels. Other types of equalizers have therefore been proposed, especially ones with a nonlinear structure denoted as decision feedback equalizer (DFE), where, after a first transversal filter aiming at reducing the precursors of the equivalent pulse at the detection point, a linear feedback filter, whose input is the sequence of past detected data symbols, removes by cancellation the intersymbol interference due to postcursors. Hence, the structure is nonlinear with respect to the received signal. Indeed, due to the feedback of detected data symbols, the DFE is hard to analyze. However in general, its performance is much better than that of a linear equalizer and can come close to that of an optimum sequence detector, e.g., implemented by the Viterbi algorithm, for a much lower complexity [2].

The signal processing complexity (number of arithmetic operations per data symbol) in time domain equalization, exemplified by the number of transversal filter tap coefficients, increases at least linearly with the number of data symbol intervals spanned by the channel impulse response. Frequency domain processing of blocks of signals, using discrete Fourier transforms (DFT), provides lower complexity per data symbol, and has therefore recently emerged as the preferred mitigation approach to channel frequency selectivity, for next-generation broadband wireless systems with bit rates of tens or hundreds of megabits/s. In this overview paper, we survey frequency domain equalization structures, mostly based on the DFE principle, for single carrier wireless digital transmissions.

Serial or single carrier modulation (SCM), in which data symbols are transmitted in serial fashion, has been the traditional digital communications format since the early days of telegraphy. An alternative is multicarrier transmission, where multiple data streams, each modulating a narrowband waveform, or tone, are transmitted in parallel, thus allowing each tone to be separately equalized by a simple gain and phase factor. Multicarrier transmission has become popular and widely used within the last two decades, due mainly to its excellent complexity/ performance tradeoff for data symbol rates far above coherence bandwidths, and also for its flexible link adaptation ability [3]-[5]. Among the first military and commercial multicarrier systems were the Collins Kineplex and General Atronics KATHRYN HF radio systems [6], [7] of the 1950s and 1960s. The KATHRYN system used DFT signal processing at the transmitter and receiver. With the realization that the eigenvectors of a linear system are sinusoids, multicarrier transmission was recognized as an optimal format for frequency selective channels in the early 1960s [8], [9]. Generation and block processing of multicarrier signals in the frequency domain, are enormously simplified by implementing the DFTs by fast Fourier transforms (FFTs), as was recognized by Weinstein and Ebert in 1971 [10], yielding a signal processing complexity that grows only logarithmically with the channel impulse response length. This realization, and the ever- growing demand for higher data rates on wireless and wired systems propelled the application of multicarrier transmission to i) digital subscriber line transmission standards, where it is generally known as discrete multitone transmission, ii) IEEE 802.11a wireless LAN and iii) digital audio and video broadcast standards, where it is known as orthogonal frequency division multiplexing (OFDM), or orthogonal frequency division multiple access (OFDMA). The early success of OFDM in standards after more then twenty years since the pioneering implementations, has been marked by Bingham in his landmark paper: Multicarrier modulation for data transmission: an idea whose time has come, [3].

A related development in the early 1970s was the realization that frequency domain processing techniques could also be used to facilitate and simplify equalization of SCM systems [11]. More recently, as an alternative to the first OFDM applications in wireless standards, Sari et al. [12]-[14] pointed out that traditional SCM could enjoy an implementation simplicity/performance tradeoff similar to that of OFDM for highly frequency selective channels with the inverse DFT moved at the receiver. (A simpler structure, with applications to diversity reception, was proposed by Clark [15] a few years later.) Indeed, this is true only for a nonlinear frequency domain equalizer. In fact, only the performance of a DFE can come close to or even exceed that of OFDM [16]. SCM waveforms have the additional advantage that for a given signal power their range of amplitude, measured by the peak-to-average ratio, is significantly less than that of multicarrier signals. As a result, their transmitted spectra and performance are less affected by transmitter power amplifier nonlinearities. This allows cheaper and more efficient high power amplifiers to be used for transmitting SCM signals. A further benefit of SCM is its greater robustness to frequency offset and phase noise than that of OFDM [17] (see also [18]).

These features of robustness to radio frequency hardware impairments make single carrier with frequency domain equalization an attractive alternative to OFDM, especially for cost- and power consumption-sensitive nextgeneration wireless user terminals which transmit uplink to base stations [19]. Thus frequency domain implementations of SCM receivers can be said to be an idea whose time has come again after a hiatus of about 20 years. However the status of SCM now is not that of a potential replacement of OFDM, but rather of a complement to it. As we will see, traditional SCM can morph to a special form of multicarrier transmission, which can be called DFT-precoded OFDM. As such, it is a form of generalized multicarrier transmission [20] (see also [21] and [22]).

SCM in the form of DFT-precoded OFDM has been proposed by the European 6th framework program Wireless INitiative NEw Radio (WINNER) project as the uplink transmission format for wide area cellular scenarios, mainly on the basis of its radio frequency impairment robustness properties. WINNER downlink and local area uplink transmissions rely on OFDMA, mainly because of its flexibility 
and transmission channel adaptability properties [23]. The Third Generation Partnership Project-Long Term Evolution (3GPP-LTE) and now LTE-Advanced standards group also propose DFT-precoded OFDM, which they call single carrier frequency division multiple access for the uplink of nextgeneration wide area cellular broadband wireless systems, again with OFDMA used in the downlink [24], [25]. These initiatives and standards activities are contributing to the International Mobile Telecommunications Advanced (IMTAdvanced) initiative of the International Telecommunications Union. The $802.16 m$ Task Group of the IEEE 802.16 Wireless metropolitan area network standards group has recently been formed to contribute to IMT-Advanced. At the time of writing, its proposed standard has not been finalized, but versions of single carrier frequency domain equalization, as well as OFDM, have been considered for uplinks. The earlier 802.16a standard, which led to the WiMAX wireless metropolitan area concept, has three transmission modes: two based on versions of OFDM and one based on SCM.

The rest of the paper is organized as follows. In Section II we provide the basic principles and signal structure of SCM frequency domain nonlinear equalization. In Section III, we present various nonlinear equalization techniques implemented in the frequency domain for a single antenna system and using the direct knowledge of the channel frequency response. These structures will be extended to the case of transmitters and receivers with multiple antennas in Section IV, where we also describe an iterative equalizer fully implemented in the frequency domain. Channel estimation methods for the proposed structures are investigated in Section V. Impacts of phase noise and other disturbances on implementations of the nonlinear frequency domain equalizers are considered in Section VI. Section VII compares SCM with OFDM, with a focus of the considered nonlinear frequency domain equalization structures. Lastly, conclusions are outlined in Section VIII.

Notation: ${ }^{*}$ denotes the complex conjugate, ${ }^{\mathrm{T}}$ denotes the transpose, ${ }^{H}$ denotes the Hermitian (transpose and complex conjugate) operator. The DFT of sequence $\left\{s_{n}\right\}$, $n=0,1, \ldots, P-1$, is

$$
S_{p}=\sum_{n=0}^{P-1} s_{n} e^{-j 2 \pi \frac{n p}{P}}, \quad p=0,1, \ldots, P-1 .
$$

The inverse DFT (IDFT) of sequence $\left\{S_{p}\right\}, p=0$, $1, \ldots, P-1$, is

$$
s_{n}=\frac{1}{P} \sum_{p=0}^{P-1} S_{p} e^{j 2 \pi \frac{n p}{P}}, \quad n=0,1, \ldots, P-1 .
$$

$\boldsymbol{I}_{N}$ denotes the $N \times N$ identity matrix. Circular convolution among signals $x$ and $y$ is denoted as $(x \otimes y)$.

\section{SYSTEM DEFINITIONS AND THE FINGERPRINT OF SINGLE CARRIER FREQUENCY DOMAIN EQUALIZER: TRANSMISSION FORMAT}

A wireless mobile transmission is characterized by a slowly time-varying multipath channel between each pair of transmit and receive antennas in a multiple input-multiple output (MIMO) scenario. For a system with $N_{\mathrm{T}}$ transmit and $N_{R}$ receive antennas, we denote the impulse response of the time-invariant channel from antenna $i$ to antenna $j$ as $h_{\mathrm{Ch}}^{(j, i)}(\tau), i=1,2, \ldots, N_{\mathrm{T}}, j=1,2, \ldots, N_{\mathrm{R}}$. Upon transmission of signal $\bar{s}^{(i)}(t)$ from antenna $i$, the received signal at antenna $j$ can be written as (baseband equivalent model)

$$
\bar{r}^{(j)}(t)=\sum_{i=1}^{N_{\mathrm{T}}} \int h_{\mathrm{Ch}}^{(j, i)}(\tau) \bar{s}^{(i)}(t-\tau) d \tau+w^{(j)}(t)
$$

where $\bar{w}^{(j)}(t)$ is the noise term, which we assume to be complex Gaussian with zero mean and power spectral density $N_{0}$.

Traditionally, a SCM signal is generated as a sequential stream of data symbols, at regular time instants $n T$, for $n=\ldots, 0,1,2, \ldots$, where $T$ is the data symbol interval, and $1 / T$ is the symbol rate. Although generally receivers perform oversampling, for the sake of a simpler notation, we assume also that the received signal is filtered and sampled with rate $1 / T$. Hence we describe the transmission system by an equivalent discrete-time model where the channel is characterized by the impulse response $h_{\ell}^{(j, i)}, \ell=0,1, \ldots, N_{\mathrm{h}}-1$, obtained by sampling the cascade of the transmit filter, the channel and the receive filter. By indicating with $s_{n}^{(i)}$ the symbol transmitted from the ith antenna, the received signal after sampling can be written as

$$
r_{n}^{(j)}=\sum_{i=1}^{N_{\mathrm{T}}} \sum_{\ell=0}^{N_{\mathrm{h}}-1} h_{\ell}^{(j, i)} s_{n-\ell}^{(i)}+w_{n}^{(j)}
$$

where $w_{n}^{(j)}$ is the noise term with variance $\sigma_{w}^{2}$

In order to allow frequency domain block equalization of the received signal, the convolutions in (4) must be circular and this can be achieved in different ways.

As we will first consider the single input-single output case, we drop the antenna index for sake of a simpler notation. The MIMO case is considered in Section IV.

\section{A. Circular and Linear Convolution}

The transmitted signal $\left\{s_{n}\right\}$ depends on the information signal $\left\{d_{n}\right\}$ but, in general, the two may not coincide. We examine conditions such that each linear convolution in (4) appears as a circular convolution between the 
channel impulse response and the information data signals $d_{n}$.

Let us consider the sequence of data symbols in blocks of $M,\left\{d_{n}\right\}, n=0,1, \ldots, M-1$, and the $N_{\mathrm{h}}$-size sequence $\left\{h_{n}\right\}, n=0,1, \ldots, N_{\mathrm{h}}-1$, with $M>N_{\mathrm{h}}$. We define the periodic signals of period $P, d_{\mathrm{rep}_{P}, n}=d_{(n \bmod P)}$, and $h_{\text {rep }_{P}, n}=h_{(n \bmod P)}, n=0,1, \ldots, P-1$, where in order to avoid time aliasing, $P \geq M$ and $P \geq N_{\mathrm{h}}$.

Now, the circular convolution between $\left\{d_{n}\right\}$ and $\left\{h_{n}\right\}$ is a periodic sequence of period $P$ defined as

$$
x_{n}^{(\text {circ })}=(h \otimes d)_{n}=\sum_{\ell=0}^{P-1} h_{\mathrm{rep}_{p}, n-\ell} d_{\mathrm{rep}_{p}, \ell} .
$$

Then, if we indicate with $\left\{D_{p}\right\},\left\{H_{p}\right\}$ and $\left\{X_{p}^{(\text {(circ })}\right\}$, $p=0,1, \ldots, P-1$, the $P$-point DFT of sequences $\left\{d_{n}\right\}$, $n=0,1, \ldots, P-1,\left\{h_{n}\right\}$, and $\left\{x_{n}^{(\text {circ })}\right\}, n=0,1, \ldots, P-1$, respectively, we obtain

$$
X_{p}^{(\text {circ })}=H_{p} D_{p}, \quad p=0,1, \ldots, P-1 .
$$

The linear convolution with support $n=0,1, \ldots, M+$ $N_{\mathrm{h}}-2$ is

$$
x_{n}^{(\operatorname{lin})}=\sum_{\ell=0}^{N_{\mathrm{h}}-1} h_{\ell} d_{n-\ell} .
$$

By comparing (7) with (5), it is easy to see that only if $P \geq M+N_{\mathrm{h}}-1$, then

$$
x_{n}^{(\operatorname{lin})}=x_{n}^{(\text {circ })}, \quad n=0,1, \ldots, P-1 .
$$

To compute the convolution between the two finite-length sequences $\left\{d_{n}\right\}$ and $\left\{h_{n}\right\},(8)$ requires that both sequences be completed with zeros (zero padding) to get a length of $P=M+N_{\mathrm{h}}-1$ samples. Then, taking the $P$-point DFT of the two sequences, performing the product (6), and taking the inverse transform of the result, one obtains the desired linear convolution.

However, there are other conditions, some of which are listed below, that yield a partial equivalence between the circular convolution $\left\{x_{n}^{(\text {(circ) }}\right\}$ and the linear convolution

$$
x_{n}=\sum_{\ell=0}^{N_{\mathrm{h}}-1} h_{\ell} s_{n-\ell},
$$

where $\left\{s_{n}\right\}$ depends on $\left\{d_{n}\right\}$.
Overlap and Save: We consider as the transmitted signal $s_{n}=d_{n}, n=0,1, \ldots, M-1$ and assume $P=M$. We verify that (9) coincides with (5) only for the instants $n=N_{h}-1$, $N_{\mathrm{h}}, \ldots, M-1$, [26]. In other words, the equivalence between the linear and the circular convolution holds always on a subset of the computed points.

Cyclic Prefix: An alternative to overlap and save is to consider, instead of the transmission of the data sequence $\left\{d_{n}\right\}$, an extended sequence $\left\{s_{n}\right\}$ that is obtained by partially repeating $\left\{d_{n}\right\}$ with a cyclic prefix of $L \geq N_{\mathrm{h}}-1$ samples, [26]:

$$
s_{n}= \begin{cases}d_{n} & n=0,1, \ldots, M-1 \\ d_{M+n} & n=-L, \ldots,-2,-1\end{cases}
$$

Moreover, assume $P=M$. It is easy to prove that (9) coincides with (5) for $n=0,1, \ldots, M-1$. Moreover, the equivalence (6) in the frequency domain holds for DFTs of size $P=M$, the data block size. This arrangement is used also in multicarrier communications [11].

Pseudo Noise (PN) Extension: Consider a sequence $\left\{s_{n}\right\}$, obtained by $\left\{d_{n}\right\}$ with the addition of a fixed sequence $p_{n}$, $n=0,1, \ldots, L-1$, of $L \geq N_{\mathrm{h}}-1$ samples, i.e.,

$$
s_{n}= \begin{cases}d_{n} & n=0,1, \ldots, M-1 \\ p_{n-M} & n=M, \ldots, M+L-1 .\end{cases}
$$

The first data block is also preceded by the sequence $\left\{p_{n}\right\}$. Moreover, now $P=M+L$. The sequence $\left\{p_{n}\right\}$ can contain any symbol sequence, including all zeros (zero padding) [27], [28], or a PN symbol sequence, denoted PN extension or unique word. The choice of the extension is also influenced by other factors, such as channel estimation [29]. It can be easily proved, that (9) coincides with $(h \otimes s)_{n}$ for $n=0,1, \ldots, P-1$, where now the circular convolution is on $s_{n}$ instead of $d_{n}$.

With reference to the noisy MIMO scenario (4), we can organize the transmitted signal $\left\{s_{n}\right\}$ into blocks of size $P$, each obtained by extending with a PN sequence a data block of size $M$. Moreover, at the beginning a PN sequence is transmitted first. Let $\left\{s_{n+k P}\right\}, n=0,1, \ldots, P-1$ be the $k$ th block and let $\left\{H_{p}^{(j, i)}\right\}$ be the $P$-size DFT of the channel impulse response $\left\{h_{\ell}^{(j, i)}\right\}$. Then we obtain

$$
\begin{aligned}
R_{p}^{(j)}(k)=\sum_{i=1}^{N_{\mathrm{T}}} H_{p}^{(j, i)} S_{p}^{(i)}(k)+W_{p}^{(j)}(k) & \\
r & =0,1, \ldots, P-1
\end{aligned}
$$

where $W_{p}^{(j)}(k)$ is the noise term in the frequency domain, which according to the hypothesis on $\left\{w_{n}\right\}$ is i.i.d. with 
variance $\sigma_{W}^{2}=P \sigma_{w}^{2}$. Note that in this arrangement the DFTs are of size $P=M+L$ instead of size $M$ as in the cyclic prefix arrangement. Moreover, in this arrangement, an additional PN extension is required before the first data block. Among the advantages of this format are a simple channel estimation, by using the PN sequence [29], and the possibility of implementing an efficient frequency domain (FD) nonlinear equalizer, as detailed in Section III. Generally, the PN extension yields a reduced bit error rate with respect to the cyclic prefix, since in the latter case data detection errors affect both the information data and the cyclic prefix, thus reducing the intersymbol interference cancellation capabilities of the nonlinear equalizer. In the following we will consider operations on a single data block and we will drop the index $k$ from FD signals.

\section{B. Signal Generation}

As described in the previous section, the data symbol sequence may be organized into DFT blocks, which may include PN extensions, or to which cyclic prefixes are appended, thus facilitating DFT processing and FD equalization at the receiver. The resulting data sequences, with or without extensions and prefixes, are low pass filtered for bandlimiting and spectrum-shaping purposes, before being up-converted to the carrier frequency.

Fig. 1 shows a generalized multicarrier transmitter architecture [19], [20], [22], which can be adapted to generate a wide variety of signals, including SCM signals, as well as OFDM, OFDMA, multicarrier code division multiple access (CDMA), etc. Because its processing occurs in the FD, it is easy to generate signals with arbitrary spectra, and to insert FD pilot tones for channel estimation (see Section V). Complexity is not a major issue since processing is done with DFTs and IDFTs, implemented by FFTs. In the figure, the IDFT block is preceded by a general pre-matrix operation, which may include a DFT, spreading, a selection mechanism and/or an allocation to multiple transmitting antennas in a MIMO or space-time code. Recognition of this generalized structure can also be found in [30]-[32].

Generation of a SCM signal block proceeds as follows. After coding and serial to parallel (S/P) conversion, blocks of $N$ coded data symbols are mapped to the FD by a $N$-point DFT. The resulting FD data components are mapped by the pre-matrix time-frequency-space selector to a set of $M \geq N$ data-carrying subcarriers, and then processed by a $M$-point inverse DFT to convert back to the time domain (TD). The resulting samples are parallel-to-serial (P/S) converted and appended with a prefix or extension for transmission. The simplest frequency mapping is to $N$ contiguous subcarrier frequencies, with the remaining $M-N$ being padded with zeroes. In this case, the output samples are expressed as

$$
\begin{aligned}
s_{n} & =\frac{1}{M} \sum_{\ell=0}^{N-1} d_{\ell} \sum_{p=0}^{N-1} e^{j 2 \pi \frac{p\left(n-\ell \frac{M}{N}\right)}{M}} \\
& =\sum_{\ell=0}^{N-1} g\left(n-\ell \frac{M}{N}\right) d_{\ell}, \quad n=0,1, \ldots, M-1
\end{aligned}
$$

where

$$
g(n)=e^{j 2 \pi \frac{(N-1) n}{M}} \frac{1}{M} \frac{\sin \left(\frac{\pi N n}{M}\right)}{\sin \left(\frac{\pi n}{M}\right)}
$$

while $\left\{s_{n}\right\}, n=-L,-L+1, \ldots,-1$, contains the cyclic prefix.

This is recognized as a block of data symbols serially transmitted at intervals of $M / N$ samples. The sampled pulse waveform given by (14) is a circular version of a sinc pulse with zero excess bandwidth, limited to a bandwidth $N / M T$. SCM signals generated in this way are called DFTprecoded OFDM signals by the WINNER project [23], and local single carrier FDMA (SC-FDMA) by the 3GPP-LTE standards body [24], [25]. For (13), $s_{m M / N}=N / M d_{m}$, thus the DFT-precoded OFDM waveform at data symbol intervals depends only on a single data symbol, and therefore has a significantly lower peak to average power ratio than that of a corresponding OFDM waveform, whose sample

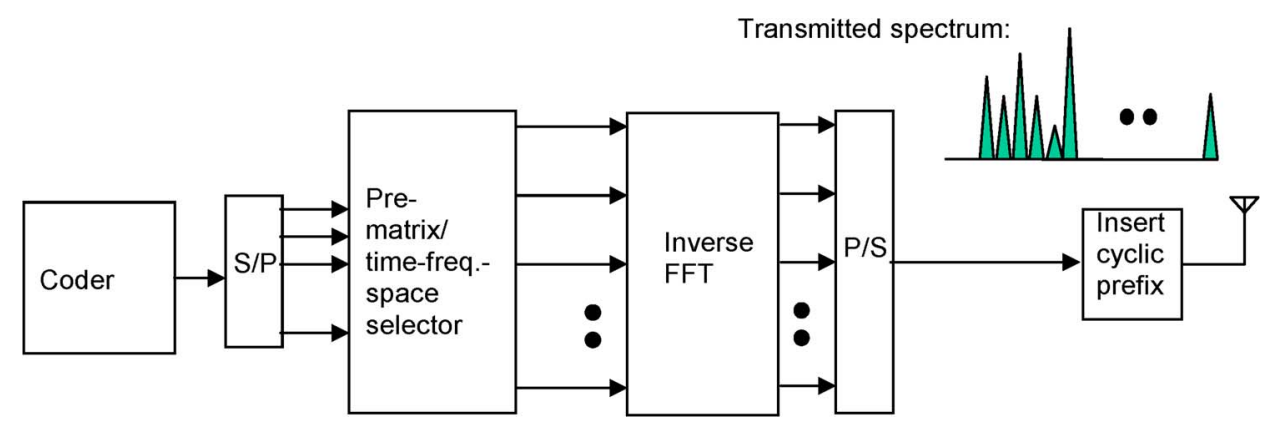

Fig. 1. Generalized multicarrier transmitter (from [22]). 